\title{
Effects of nitrogen and phosphorus on the abundance and cell size of planktonic nanoflagellate communities
}

\author{
Efeito da concentração de nitrogênio e fósforo na abundância e \\ tamanho celular da comunidade de nanoflagelados planctônicos
}

Danielle Goeldner Pereira ${ }^{1}$, Fábio Amodêo Lansac-Tôha ${ }^{1}$, Gustavo Mayer Pauleto ${ }^{1}$, Luis Mauricio Bini ${ }^{2}$ and Luiz Felipe Machado Velho ${ }^{1}$

${ }^{1}$ Núcleo de Pesquisas em Limnologia, Ictiologia e Aqüicultura - NUPÉLIA, Universidade Estadual de Maringá - UEM, Av. Colombo, 5790, CEP 87020-900, Maringá, PR, Brazil e-mail: daniellegp80@gmail.com; fabio@nupelia.uem.br; gmayer@hotmail.com; felipe.velho@gmail.com

${ }^{2}$ Departamento de Ecologia, Instituto de Ciências Biológicas - ICB, Universidade Federal de Goiás - UFG, CEP 74001-970, Goiânia, GO, Brazil e-mail:1mbini@gmail.com

\begin{abstract}
Aim: We experimentally investigated the effects of nutrients (Nitrogen and Phosphorus) enrichment on the density, biomass, and cell size of pigmented and heterotrophic plankton nanoflagellates communities. Methods: The experiment was done in mesocosms in a tropical reservoir during a 19-day period. Four different treatments were carried out: Control (non-nutrient addition - $\mathrm{C}$ ), phosphorus additions $(\mathrm{P})$, nitrogen addition $(\mathrm{N})$ and phosphorus + nitrogen addition $(\mathrm{N}+\mathrm{P})$. Each treatment was performed in triplicate, sorted randomly, thus giving a total of 12 experimental carboys, which were placed transversely in the middle of the reservoir. Results: In general, pigmented and heterotrophic nanoflagellates fractions responded to nutrient addition, increasing densities and biomass values at the fertilized treatments. Opposed to expected, enriched treatments resulted in a slight decrease in mean cell size of the pigmented fraction. Moreover, in nutrient-rich treatments, pigmented nanoflagellates had higher relative abundance than in the control. Conclusions: Our results indicate that: i) the density and biomass of nanoflagellates responded to the nutrient enrichment, mainly when $\mathrm{N}$ and $\mathrm{P}$ were added together; ii) the pigmented and heterotrophic fractions showed distinct time responses to fertilization; iii) the growth of nanoflagellate community seems to be co-limited by $\mathrm{N}$ and $\mathrm{P}$; iv) the nutrient enrichment led to a greater pigmented than heterotrophic fraction contribution; and v) among the analyzed variables, nanoflagellate densities seem to be more sensitive to changes in nutrient availability than biomass or mean cell size.
\end{abstract}

Keywords: plankton, protozoa, fertilization, mesocoms.

Resumo: Objetivo: Investigamos experimentalmente o efeito da adição de nutrientes (Nitrogênio e Fósforo) sobre a densidade e o tamanho celular da comunidade de nanoflagelados planctônicos pigmentados e heterotróficos. Métodos: $\mathrm{O}$ experimento foi desenvolvido em mesocosmos num reservatório tropical durante 19 dias. Quatro diferentes tratamentos foram utilizados: Controle (sem adição de nutrientes - C), adição de fósforo $(\mathrm{P})$, adição de nitrogenio $(\mathrm{N})$ e adição de fósforo + nitrogênio $(\mathrm{N}+\mathrm{P})$. Cada tratamento foi realizado em triplicata, sorteado randomicamente, totalizando 12 unidades experimentais as quais foram instaladas transversalmente no meio do reservatório. Resultados: Em geral, os nanoflagelados pigmentados e heterotróficos responderam à adição de nutrientes, com incremento na densidade e biomassa nos tratamentos fertilizados. Ao contrário do esperado, os tratamentos enriquecidos mostraram um leve decréscimo no tamanho celular médio da fração pigmentada. Além disso, a contribuição relativa dos nanoflagelados pigmentados para a abundancia total foi maior nos tratamentos fertilizados quando comparada ao controle. Conclusão: Nossos resultados indicaram que: i) a densidade e a biomassa dos nanoflagelados responderam ao enriquecimento por nutrientes, principalmente quando $\mathrm{N}$ e $\mathrm{P}$ foram adicionados em conjunto; ii) as frações pigmentadas e heterotróficas apresentaram tempos distintos de resposta a fertilização; iii) o crescimento da comunidade de nanoflagelados parece ser co-limitada por N e P; iv) o enriquecimento por nutrientes tornou a contribuição da fraçáo pigmentada maior que a contribuição da fração heterotrófica; e v) entre as variáveis analisadas da comunidade de nanoflagelados, a densidade pareceu ser mais sensível às alteraçóes na disponibilidade de nutrientes quando comparada a biomassa e ao tamanho celular médio.

Palavras-chave: plâncton, protozoários, fertilização, mesocosmos. 


\section{Introduction}

The effects of nutrient fertilization and the importance of bottom-up control on aquatic communities are both current subjects of discussion (Auer et al., 2004; Samuelsson et al., 2006). The bottom-up hypothesis proposes that resources regulate community structure, and that any increase in resources results in a subsequent increase in algal biomass production, followed by biomass increases of higher trophic levels (Lampert and Sommer, 1997).

Studies on planktonic communities have shown that eutrophication leads to an increase not only in the abundance of classical grazing food web, but also affects the microbial food web components such as bacteria and protozoan communities (Sipura et al., 2005; Pagioro et al., 2005; Samuelsson et al., 2006). Many experimental studies carried out in temperate region have shown that nanoflagellates $(2-20 \mathrm{~mm})$ respond positively to the increase in available nutrients (Jansson et al., 1996; Gilbert et al., 1998; Samuelsson et al., 2002; Simek et al., 2003). However, a recent study by Domènech et al. (2006) showed that nanoflagellates did not respond significantly to fertilization, suggesting that the increase of nanoflagellate abundance to fertilization is not a general pattern for different ecosystems.

Regarding the nutrient effects on the abundance of different fractions of plankton communities, some studies observed that, in general, the autotrophic fraction dominated at oligotrophic conditions, and the heterotrophic contribution increased with increase in trophic status of the ecosystems (Auer et al., 2004; Samuelsson et al., 2006). Nutrient enrichment can also affects the size structure of communities. Specifically, more resources lead to a replacement of smaller by bigger individuals in distinct fractions of microbial communities (Kress et al., 2005; Sabetta et al., 2005; Sipura et al., 2005; Samuelsson et al., 2006).

Another important issue in aquatic ecology refers to the main limiting nutrient for the community development. In tropical regions, there are controversies about the main limiting nutrient, nitrogen $(\mathrm{N})$ or phosphorus $(\mathrm{P})$, to the aquatic productivity. Recently, phosphorus was evidenced as the main limiting nutrient to primary production (Carvalho et al., 2003; Rejas et al., 2005). However, Lewis Junior (2000) showed productivity limited by nitrogen whereas Huszar (2006) suggested that systems can be co-limited by $\mathrm{N}$ and $\mathrm{P}$, without showing a uniform nutrient limitation.
In this study, we carried out an experiment to measure the effects of nitrogen and phosphorus additions on the density, biomass and size structure of the nanoflagellate community from a tropical reservoir. We predicted that $\mathrm{N}$ and $\mathrm{P}$ fertilization would lead to an increase in density, mean cell size, and biomass values of the pigmented (PNF) and heterotrophic (HNF) nanoflagellates. We also predicted that $\mathrm{N}$ and $\mathrm{P}$ additions would increase the contribution of the heterotrophic fraction to the total biomass and density of nanoflagellate community. Lastly, we hypothesized that phosphorus is the main nutrient limiting the growth of these populations in this environment.

\section{Material and Methods}

\subsection{Experimental site}

The mesocosm experiment was carried out in the Corvo River, a tributary of the Rosana Reservoir. The site presents oligo-mesotrophic conditions (Roberto et al., 2005) and is located along the lower stretch of the Paranapanema River in Paraná State, Brazil (22 $36^{\prime} \mathrm{S}$ and $\left.52^{\circ} 50^{\prime} \mathrm{W}\right)$ (Figure 1).

\subsection{Experimental design}

The experiment was done in mesocoms during a 19 day period, between November and December 2004. Four different treatments were carried out: Control (non-nutrient addition - C), phosphorus additions $(\mathrm{P})$, nitrogen addition $(\mathrm{N})$ and phosphorus + nitrogen addition $(\mathrm{N}+\mathrm{P})$. Each treatment was performed in triplicate, sorted randomly, thus giving a total of 12 experimental carboys, which were placed 1 meter distant from each other in a transversely line in the middle of the reservoir. The mesocosms utilized were made with polyethylene bags ( $1 \mathrm{~m}$ deep, with $1 \mathrm{~m}^{3}$ volume) and remained opened on the top, isolated from the sediment and suspended by buoys. They were filled at the beginning of the experiment with water from the reservoir (sampled at subsurface), without any previous treatment. In order to increase natural concentrations $\left(3.16 \pm 0.18 \mathrm{mmol}\right.$ of $\mathrm{KNO}_{3}$ and $0.0882 \pm 0.019 \mathrm{mmol}$ of $\left.\mathrm{KH}_{2} \mathrm{PO}_{4}\right)$ in the $\mathrm{P}, \mathrm{N}$ and $\mathrm{N}+\mathrm{P}$ treatments, we added $9.89 \mu \mathrm{mol}$ of $\mathrm{KNO}_{3}$ and $0.29 \mu \mathrm{mol}$ of $\mathrm{KH}_{2} \mathrm{PO}_{4}$ at the beginning of the experiment.

\subsection{Sampling schedule and methods}

Subsurface samples $(50 \mathrm{~cm}$ depth) for abiotic and biotic measurements were daily taken until the 


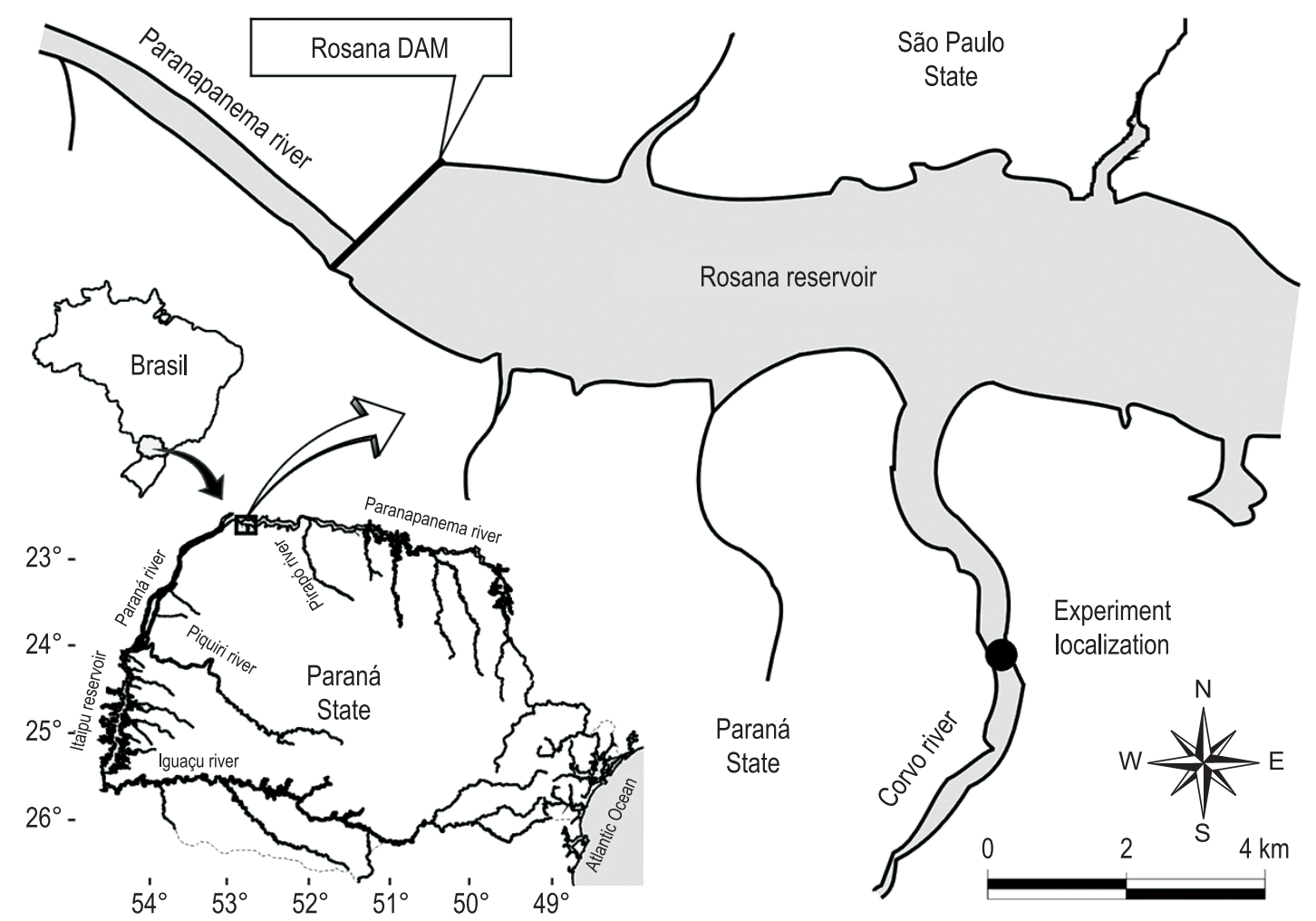

Figure 1. Rosana Reservoir and the location of the mesocosm experiments.

$4^{\text {th }}$ experimental day, every two days until the $16^{\text {th }}$ and on the $19^{\text {th }}$ experiment day.

Limnological variables samples taken directly inside the mesocosms were: temperature $\left({ }^{\circ} \mathrm{C}\right)$, oxigen concentration $\left(\mathrm{mg} \mathrm{L}^{-1}\right)(\mathrm{YSI})$, conductivity $\left(\mathrm{mS} \mathrm{cm}{ }^{-1}\right)$ and $\mathrm{pH}$ (Digimed).

The water to measure ciliate abundance $(1 \mathrm{~L})$, total phosphorus, total nitrogen and chlorophyll- $a$ concentration (1 L) was collected using plastic bottles and preserved in the cooler until later laboratorial procedure.

Samples to establish bacteria and nanoflagellate density and biomass were sampled with bottle glass $(100 \mathrm{~mL})$ and preserved with a solution of alkaline Lugol, formalin and sodium thiosulfate (Sherr and Sherr, 1993).

The zooplankton community samples (cladocerans, copepods and rotifers) consisted of $20 \mathrm{~L}$ of water collected using a plastic bucket. The water sampled was filtered through a $68 \mu \mathrm{m}$ mesh plankton net and preserved immediately with buffered formalin (4\%).

Analyses of total phosphorus (P-total-mg L ${ }^{-1}$ ) (Mackereth et al., 1978), total nitrogen (N-Total$\mathrm{mg} \mathrm{L}^{-1}$ ) (Bergamin et al., 1978) and chlorophyll-a concentration $\left(\mu \mathrm{g} \mathrm{L}^{-1}\right)$ (Golterman et al., 1978) were done in the laboratory.
For bacteria enumeration $(0.2-2 \mu \mathrm{m})$ and nanoflagellates $(2-20 \mathrm{~mm})$, water samples were stained with DAPI (4,6' - diamidino-2phenylindole: $0.001 \%$ final cons). Concentration was done by gently vacuum filtration $(<15 \mathrm{~cm}$ of $\mathrm{Hg}$ ) on to $0.2 \mu \mathrm{m}$ (for bacteria) and $0.8 \mathrm{~mm}$ (for nanoflagellates) Nucleopore polycarbonate membranes. The volume filtered was in the range 0.1-15 mL. Between 400 bacteria or 50 fields and 100-300 nanoflagellates or 100 fields per sample, randomly distributed on the filter, were counted with Olympus epifluorescence microscope at $1000 \times$ magnification. Total bacteria and nanoflagellates abundance was measured by UV light (white-blue fluorescence). Pigmented and heterotrophic nanoflagellates were differentiated by filter set which provided blue excitation (resulting in red autofluorescence by pigmented and green fluorescence by heterotrophic organisms). HNF abundance was the difference between total nanoflagellates abundance and PNF.

The individual cell volume of each measured nanoflagellate was derived from mean cell size estimated based on the largest dimension of each cell and the approximated geometric shape (Wetzel and Likens, 1991). Cell biovolume data were converted 
to carbon content using the expression proposed by Fenchel (1982) (Equation 1):

$1 \mu \mathrm{m}^{3}=167 \mathrm{fg}$ C.

Considering ciliate density, the samples of $1000 \mathrm{~mL}$ were concentrated to $100 \mathrm{~mL}$ by filtering the water through $12 \mu \mathrm{m}$ mesh plankton net. Subsequently, the organisms were analyzed in vivo in optic microscopy Olympus CX41 at 100x and $400 \times$ by assessing sub-samples of 10 aliquots of $100 \mu \mathrm{L}$ from the concentrated sample. The ciliates density was expressed as ind. $\mathrm{L}^{-1}$.

Zooplankton counting was based on the methodology by Bottrell et al. (1976), in which three subsamples from each sample were analyzed. The abundance was expressed as individuals per $\mathrm{m}^{-3}$.

\subsection{Statistical analysis}

In order to analyze the effects of nutrient enrichment ( $\mathrm{P}$ and $\mathrm{N}$ ) on nanoflagellate density, biomass, cell size, and the ratio PNF:HNF (in terms of both biomass and density), a repeated measure Analysis of Variance (ANOVAR) was performed. Moreover, data were tested regarding homocedasticity (Levene's test) and sphericity
(Mauchley's test). These analyses were performed using STATISTICA, version 5.0 (StatSoft, 1997). To equalize the variance in all statistical analyses the variables were previously transformed $\log (\mathrm{x}+1)$.

\section{Results}

\subsection{Nutrients}

A similar temporal pattern of a decrease of nitrogen concentration was observed between control and nutrient addition treatments after the $3^{\text {rd }}$ day. However, higher mean values were registered after nutrient addition, with mean values between 206.3 and $1434 \mu \mathrm{g} \mathrm{L}^{-1}$ along the experiment (Table 1). As observed for nitrogen, an increase on the mean values of phosphorus was registered after nutrient addition. Phosphorus mean values varied, respectively, between 7.1 and $57 \mu \mathrm{g} \mathrm{L}^{-1}$, and between 0.6 and $21.5 \mu \mathrm{g} \mathrm{L}^{-1}$ (Table 1).

\subsection{Nanoflagellate density}

Nutrient enrichment had a positive effect on the mean density of PNF and HNF (Figure 2). PNF and HNF densities varied, respectively, between $0.59 \times 10^{2}$ cells $\mathrm{mL}^{-1}$ at the beginning of
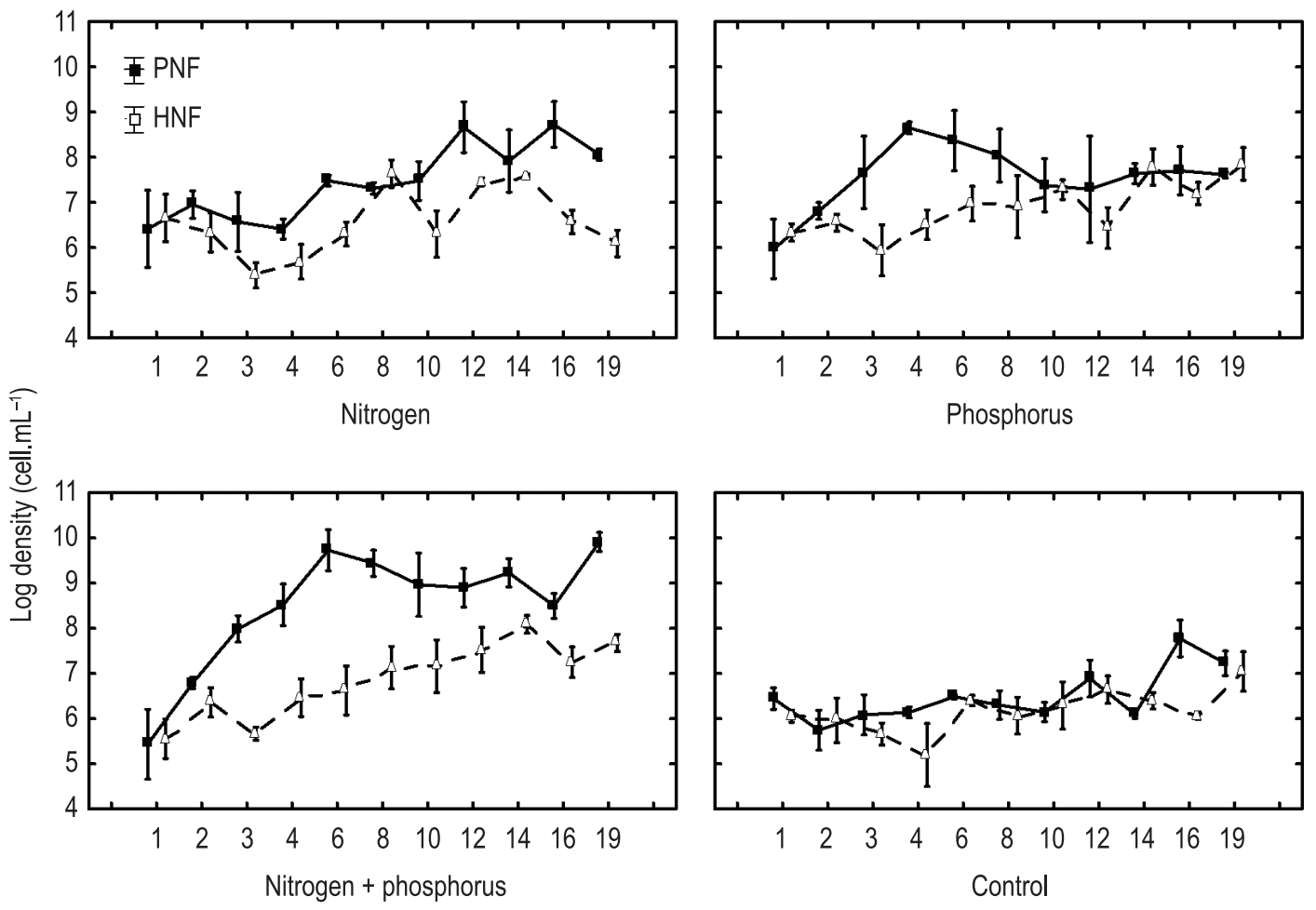

Days

Figure 2. Variation of pigmented (PNF) and heterotrophic (HNF) nanoflagellate densities during 19 days of a nutrient addition experiment. Data shown are mean \pm standard deviation. 
Table 1. Data of abiotic and biotic variables (mean and standard deviation) of the experiment carried out in the Corvo River.

\begin{tabular}{|c|c|c|c|c|}
\hline Variables/Days & Nitrogen & Phosphorus & $\begin{array}{c}\text { Nitrogen+ } \\
\text { Phosphorus }\end{array}$ & Control \\
\hline $\begin{array}{c}\text { Chlorophyll-a } \\
\left(\mu \mathrm{g} . \mathrm{L}^{-1}\right)\end{array}$ & $\begin{array}{c}\text { Mean/Standard } \\
\text { deviation }\end{array}$ & $\begin{array}{c}\text { Mean/Standard } \\
\text { deviation }\end{array}$ & $\begin{array}{c}\text { Mean/Standard } \\
\text { deviation }\end{array}$ & $\begin{array}{c}\text { Mean/Standard } \\
\text { deviation }\end{array}$ \\
\hline 1 & $1.1( \pm 0.5)$ & $0.7( \pm 0.3)$ & $0.7( \pm 0.3)$ & $2.0( \pm 1.6)$ \\
\hline 2 & $1.3( \pm 0.3)$ & $0.6( \pm 0)$ & $0.7( \pm 0.3)$ & $0.9( \pm 0.3)$ \\
\hline 3 & $1.3( \pm 0.3)$ & $2.5( \pm 1.1)$ & $3.6( \pm 1.8)$ & $0.6( \pm 0)$ \\
\hline 4 & $0.7( \pm 0.3)$ & $2.4( \pm 0.3)$ & $4.9( \pm 2.5)$ & $1.3( \pm 0.8)$ \\
\hline 6 & $0.8( \pm 0.4)$ & $3.0( \pm 1.0)$ & $21.5( \pm 8.3)$ & $0.9( \pm 0.2)$ \\
\hline 8 & $1.5( \pm 0.6)$ & $2.0( \pm 2.4)$ & $12.5( \pm 4.3)$ & $0.7( \pm 0.3)$ \\
\hline 10 & $1.6( \pm 1.2)$ & $2.2( \pm 0.8)$ & $9.4( \pm 6.5)$ & $1.3( \pm 0.6)$ \\
\hline 12 & $2.2( \pm 1.1)$ & $1.8( \pm 0.8)$ & $6.9( \pm 0.6)$ & $1.8( \pm 0.3)$ \\
\hline 14 & $3.8( \pm 2.5)$ & $3.3( \pm 0.5)$ & $5.8( \pm 1.7)$ & $1.3( \pm 0.3)$ \\
\hline 16 & $3.6( \pm 0.3)$ & $2.7( \pm 1.4)$ & $6.9( \pm 1.1)$ & $1.8( \pm 0.3)$ \\
\hline 19 & $2.4( \pm 0.8)$ & $2.4( \pm 1.1)$ & $6.2( \pm 1.1)$ & $1.8( \pm 0.3)$ \\
\hline \multicolumn{5}{|c|}{ Total phosphourus $\left(\mu \mathrm{g} \cdot \mathrm{L}^{-1}\right)$} \\
\hline 1 & $8.1( \pm 2.3)$ & $10.2( \pm 3.6)$ & $7.7( \pm 0.5)$ & $7.1( \pm 1.2)$ \\
\hline 2 & $11.3( \pm 0.6)$ & $47.0( \pm 1.1)$ & $46.5( \pm 2.9)$ & $9.8( \pm 1.7)$ \\
\hline 3 & $14.1( \pm 1.1)$ & $43.1( \pm 2.4)$ & $44.5( \pm 1.0)$ & $13.4( \pm 1.3)$ \\
\hline 4 & $28.7( \pm 2.1)$ & $43.1( \pm 1.9)$ & $57.0( \pm 3.0)$ & $28.2( \pm 3.0)$ \\
\hline 6 & $16.2( \pm 0.7)$ & $43.1( \pm 3.4)$ & $41.0( \pm 2.7)$ & $14.5( \pm 1.3)$ \\
\hline 8 & $15.1( \pm 0.9)$ & $43.1( \pm 2.4)$ & $41.5( \pm 6.5)$ & $15.9( \pm 2.2)$ \\
\hline 10 & $16.8( \pm 1.1)$ & $43.1( \pm 0.6)$ & $40.4( \pm 6.5)$ & $17.8( \pm 0.6)$ \\
\hline 12 & $16.3( \pm 1.4)$ & $43.1( \pm 2.2)$ & $36.7( \pm 4.9)$ & $17.3( \pm 0.8)$ \\
\hline 14 & $16.8( \pm 2.5)$ & $43.1( \pm 2.5)$ & $33.6( \pm 3.1)$ & $19.9( \pm 2.1)$ \\
\hline 16 & $14.0( \pm 0.3)$ & $43.1( \pm 2.4)$ & $32.2( \pm 0.7)$ & $17.6( \pm 1.3)$ \\
\hline 19 & $16.2( \pm 2.0)$ & $28.4( \pm 1.4)$ & $24.7( \pm 7.1)$ & $17.0( \pm 2.1)$ \\
\hline \multicolumn{5}{|c|}{ Total nitrogen $\left(\mu \mathrm{g} \cdot \mathrm{L}^{-1}\right)$} \\
\hline 1 & $329.0( \pm 25.3)$ & $305.8( \pm 17.8)$ & $312.5( \pm 13.4)$ & $334.5( \pm 7.1)$ \\
\hline 2 & $1194.1( \pm 33.8)$ & $280.9( \pm 36.8)$ & $1068.5( \pm 58.6)$ & $345.6( \pm 14.7)$ \\
\hline 3 & $1434.6( \pm 112.4)$ & $275.8( \pm 31.6)$ & $1168.6( \pm 85.4)$ & $384.0( \pm 1.5)$ \\
\hline 4 & $1199.7( \pm 198.4)$ & $254.6( \pm 28.6)$ & $1004.3( \pm 32.2)$ & $352.6( \pm 17.4)$ \\
\hline 6 & $980.4( \pm 213.5)$ & $227.1( \pm 10.9)$ & $592.7( \pm 67.5)$ & $239.4( \pm 5.5)$ \\
\hline 8 & $905.7( \pm 192.1)$ & $255.5( \pm 51.8)$ & $629.5( \pm 34.1)$ & $221.3( \pm 4.0)$ \\
\hline 10 & $799.0( \pm 172.2)$ & $284.1( \pm 43.6)$ & $555.6( \pm 114.6)$ & $281.1( \pm 5.7)$ \\
\hline 12 & $678.7( \pm 290.9)$ & $163.4( \pm 13.6)$ & $509.2( \pm 157)$ & $212.6( \pm 14.1)$ \\
\hline 14 & $587.4( \pm 194.0)$ & $287.2( \pm 56.4)$ & $457.8( \pm 102.3)$ & $221.9( \pm 20.5)$ \\
\hline 16 & $432.2( \pm 187.2)$ & $198.8( \pm 34.7)$ & $387.2( \pm 47.4)$ & $206.3( \pm 10.2)$ \\
\hline 19 & $431.0( \pm 121.9)$ & $281.5( \pm 37.1)$ & $361.0( \pm 50.6)$ & $238.6( \pm 7.2)$ \\
\hline \multicolumn{5}{|c|}{ Total conductivity $\left(\mu \mathrm{S} . \mathrm{cm}^{-1}\right)$} \\
\hline 1 & $36.2( \pm 0.5)$ & $36.3( \pm 0.1)$ & $36.4( \pm 0.4)$ & $36.6( \pm 0.3)$ \\
\hline 2 & $47.4( \pm 0.9)$ & $36.8( \pm 0.05)$ & $47.3( \pm 0.3)$ & $36.9( \pm 0.1)$ \\
\hline 3 & $47.4( \pm 0.8)$ & $36.7( \pm 0.2)$ & $47.5( \pm 0.3)$ & $36.0( \pm 0.2)$ \\
\hline 4 & $47.2( \pm 2)$. & $35.2( \pm 0.05)$ & $45.3( \pm 0.3)$ & $35.5( \pm 0.4)$ \\
\hline 6 & $43.6( \pm 2.0)$ & $33.2( \pm 0.4)$ & $40.2( \pm 0.3)$ & $33.1( \pm 0.6)$ \\
\hline 8 & $40( \pm 4.3)$ & $33.2( \pm 0.5)$ & $39.5( \pm 0.5)$ & $33.2( \pm 0.3)$ \\
\hline 10 & $39.7( \pm 1.5)$ & $30.3( \pm 0.4)$ & $36.0( \pm 0.4)$ & $30.7( \pm 0.1)$ \\
\hline 12 & $42.3( \pm 1.9)$ & $32( \pm 0.3)$ & $38.9( \pm 0.1)$ & $32.2( \pm 0.2)$ \\
\hline 14 & $38( \pm 1.0)$ & $29.2( \pm 0.2)$ & $35.3( \pm 0.1)$ & $32.6( \pm 5.8)$ \\
\hline 16 & $39.9( \pm 3.2)$ & $31.6( \pm 2.2)$ & $39.1( \pm 0.05)$ & $33.6( \pm 0.6)$ \\
\hline 19 & $42.5( \pm 0.7)$ & $34.5( \pm 0.7)$ & $41.0( \pm 0.2)$ & $36.5( \pm 1.9)$ \\
\hline \multicolumn{5}{|l|}{ Total oxigen $\left(\mathrm{mg} \cdot \mathrm{L}^{-1}\right)$} \\
\hline 1 & $6.6( \pm 0.2)$ & $6.5( \pm 0.2)$ & $6.5( \pm 0.1)$ & $6.4( \pm 0.1)$ \\
\hline 2 & $6.1( \pm 0.1)$ & $4.9( \pm 0.3)$ & $4.6( \pm 0.07)$ & $6.1( \pm 0.1)$ \\
\hline
\end{tabular}

na $=$ data no avaiable. 
Table 1. Continued...

\begin{tabular}{|c|c|c|c|c|}
\hline Variables/Days & Nitrogen & Phosphorus & $\begin{array}{c}\text { Nitrogen+ } \\
\text { Phosphorus }\end{array}$ & Control \\
\hline 3 & $6.3( \pm 0.1)$ & $4.0( \pm 0.6)$ & $2.5( \pm 0.2)$ & $6.4( \pm 0.4)$ \\
\hline 4 & $5.1( \pm 0.2)$ & $3.2( \pm 0.6)$ & $1.8( \pm 0.2)$ & $4.7( \pm 0.3)$ \\
\hline 6 & $4.8( \pm 0.1)$ & $4.3( \pm 0.7)$ & $3.0( \pm 1.07)$ & $4.4( \pm 0.1)$ \\
\hline 8 & $3.3( \pm 0.3)$ & $3.8( \pm 0.8)$ & $4.0( \pm 1.7)$ & $4.0( \pm 0.3)$ \\
\hline 10 & $4.2( \pm 0.4)$ & $4.4( \pm 0.7)$ & $4.8( \pm 1.3)$ & $5.1( \pm 0.03)$ \\
\hline 12 & $3.0( \pm 0.9)$ & $3.6( \pm 0.9)$ & $2.50( \pm 0.4)$ & $4.3( \pm 0.8)$ \\
\hline 14 & $2.7( \pm 0.6)$ & $3.6( \pm 0.9)$ & $1.7( \pm 0.6)$ & $4.3( \pm 0.1)$ \\
\hline 16 & $2.3( \pm 0.5)$ & $4.1( \pm 0.2)$ & $1.6( \pm 0.7)$ & $4.2( \pm 0.2)$ \\
\hline 19 & $3.7( \pm 0.3)$ & $5.5( \pm 0.3)$ & $4.7( \pm 0.05)$ & $4.9( \pm 0.2)$ \\
\hline \multicolumn{5}{|c|}{ Water temperature $\left({ }^{\circ} \mathrm{C}\right)$} \\
\hline 1 & $25.5( \pm 0,1)$ & $24.8( \pm 1.2)$ & $25.5( \pm 0.1)$ & $25.6( \pm 0.2)$ \\
\hline 2 & $26.2( \pm 0,0)$ & $26.2( \pm 0)$ & $26.2( \pm 0.05)$ & $26.2( \pm 0.1)$ \\
\hline 3 & $27.8( \pm 0.1)$ & $27.8( \pm 0)$ & $27.8( \pm 0.05)$ & $27.8( \pm 0.05)$ \\
\hline 4 & $28.5( \pm 0)$ & $28.5( \pm 0)$ & $28.6( \pm 0.05)$ & $28.5( \pm 0.05)$ \\
\hline 6 & $26.6( \pm 0)$ & $26.6( \pm 0)$ & $26.6( \pm 0)$ & $26.6( \pm 0.0)$ \\
\hline 8 & $27.0( \pm 0)$ & $27.0( \pm 0.05)$ & $27.0( \pm 0.05)$ & $26.1( \pm 1.7)$ \\
\hline 10 & $26.2( \pm 0.05)$ & $26.2( \pm 0)$ & $26.2( \pm 0)$ & $26.2( \pm 0)$ \\
\hline 12 & $27.1( \pm 0)$ & $27.1( \pm 0)$ & $27.2( \pm 0.05)$ & $27.1( \pm 0.05)$ \\
\hline 14 & $27.7( \pm 0.05)$ & $27.7( \pm 0)$ & $27.7( \pm 0.0)$ & $27.7( \pm 0)$ \\
\hline 16 & $26.6( \pm 0.05)$ & $26.7( \pm 0)$ & $26.7( \pm 0.05)$ & $26.7( \pm 0.05)$ \\
\hline 19 & $25.5( \pm 0.05)$ & $25.4( \pm 0.1)$ & $25.5( \pm 0.05)$ & $25.5( \pm 0.05)$ \\
\hline \multicolumn{5}{|l|}{$\mathrm{pH}\left({ }^{\circ} \mathrm{C}\right)$} \\
\hline 1 & $6.8( \pm 0,06)$ & $7.0( \pm 0.5)$ & $6.7( \pm 0.03)$ & $6.7( \pm 0.06)$ \\
\hline 2 & $6.6( \pm 0,1)$ & $6.7( \pm 0.2)$ & $6.4( \pm 0.06)$ & $6.6( \pm 0.08)$ \\
\hline 3 & $6.6( \pm 0.7)$ & $6.6( \pm 0.2)$ & $6.4( \pm 0.07)$ & $6.6( \pm 0.07)$ \\
\hline 4 & $6.6( \pm 0.09)$ & $6.5( \pm 0.2)$ & $6.3( \pm 0.05)$ & $6.5( \pm 0.08)$ \\
\hline 6 & $6.6( \pm 0.07)$ & $6.5( \pm 0.09)$ & $6.6( \pm 0.1)$ & $6.6( \pm 0.01)$ \\
\hline 8 & $6.5( \pm 0.03)$ & $6.5( \pm 0.10)$ & $6.9( \pm 0.3)$ & $6.6( \pm 0.03)$ \\
\hline 10 & $6.1( \pm 0.04)$ & $6.1( \pm 0.09)$ & $6.4( \pm 0.2)$ & $6.2( \pm 0.07)$ \\
\hline 12 & $6.5( \pm 0.04)$ & $6.5( \pm 0.1)$ & $6.5( \pm 0.01)$ & $6.6( \pm 0.01)$ \\
\hline 14 & $6.2( \pm 0.04)$ & $6.1( \pm 0.08)$ & $6.1( \pm 0.05)$ & $6.3( \pm 0.02)$ \\
\hline 16 & $6.5( \pm 0.01)$ & $6.5( \pm 0.02)$ & $6.4( \pm 0.03)$ & $6.6( \pm 0.01)$ \\
\hline 19 & $6.2( \pm 0.08)$ & $6.3( \pm 0.1)$ & $6.3( \pm 0.01)$ & $6.3( \pm 0.07)$ \\
\hline \multicolumn{5}{|c|}{ Total organic carbon $\left(\mathrm{mg} \cdot \mathrm{L}^{-1}\right)$} \\
\hline 1 & $1.9( \pm 0.4)$ & $1.8( \pm 0.1)$ & $1.8( \pm 0.20)$ & $2.0( \pm 0.3)$ \\
\hline 2 & $1.4( \pm 0.03)$ & $1.5( \pm 0.2)$ & $1.5( \pm 0.08)$ & $1.4( \pm 0.1)$ \\
\hline 3 & $1.5( \pm 0.3)$ & $1.1( \pm 0.06)$ & $1.8( \pm 0.2)$ & $1.6( \pm 0.3)$ \\
\hline 4 & $1.5( \pm 0.2)$ & $1.7( \pm 0.06)$ & $2.2( \pm 0.5)$ & $2.1( \pm 0.4)$ \\
\hline 6 & $2.4( \pm 1.4)$ & $0.2( \pm 0.2)$ & $2.7( \pm 0.9)$ & $2.9( \pm 0.7)$ \\
\hline 8 & $3.0( \pm 0.4)$ & $2.9( \pm 0.2)$ & $3.6( \pm 0.2)$ & $2.4( \pm 0.3)$ \\
\hline 10 & $2.9( \pm 0.6)$ & $3.4( \pm 0.7)$ & $3.0( \pm 0.3)$ & $2.8( \pm 0.3)$ \\
\hline 12 & $3.2( \pm 0.1)$ & $3.9( \pm 0.3)$ & $4.5( \pm 0.6)$ & $3.4( \pm 0.2)$ \\
\hline 14 & $3.7( \pm 0.6)$ & $4,2( \pm 0.2)$ & $4.9( \pm 0.7)$ & $3.9( \pm 0.6)$ \\
\hline 16 & $4.1( \pm 0.2)$ & $4.2( \pm 0.3)$ & $3.5( \pm 0.5)$ & $4.8( \pm 0.7)$ \\
\hline 19 & na & na & na & na \\
\hline \multicolumn{5}{|l|}{ Bacteria (ind. $\mathrm{mL}^{-1}$ ) } \\
\hline 1 & $2088425( \pm 125030)$ & $2356998( \pm 83160)$ & $2687253( \pm 3386)$ & $1853874( \pm 6437)$ \\
\hline 2 & $2336523( \pm 35353)$ & $6724588( \pm 572987)$ & $3996422( \pm 762995)$ & $3263133( \pm 5089)$ \\
\hline 3 & $4063322( \pm 470377)$ & $5717507( \pm 929358)$ & $7254555( \pm 666798)$ & $3143557( \pm 8049)$ \\
\hline 4 & $4135163( \pm 170733)$ & $3937854( \pm 572008)$ & $4117475( \pm 82495)$ & $3270156( \pm 4166)$ \\
\hline 6 & na & na & na & na \\
\hline 8 & $3699773( \pm 295613)$ & $5858299( \pm 828340)$ & $4609003( \pm 1216547)$ & $3368543( \pm 8437)$ \\
\hline 10 & na & na & na & na \\
\hline 12 & na & na & na & na \\
\hline
\end{tabular}

na = data no avaiable. 
Table 1. Continued...

\begin{tabular}{|c|c|c|c|c|}
\hline Variables/Days & Nitrogen & Phosphorus & $\begin{array}{c}\text { Nitrogen+ } \\
\text { Phosphorus }\end{array}$ & Control \\
\hline 14 & na & na & na & na \\
\hline 16 & $3584602( \pm 297729)$ & $5721177( \pm 615503)$ & $3667632( \pm 467773)$ & $4096624( \pm 0616)$ \\
\hline 19 & na & na & na & na \\
\hline \multicolumn{5}{|l|}{ Ciliates (ind. L-1) } \\
\hline 1 & na & na & na & na \\
\hline 2 & $2.0( \pm 0.2)$ & $1.9( \pm 0.9)$ & $1.5( \pm 1.2)$ & $1.3( \pm 1.0)$ \\
\hline 3 & $2.0( \pm 0.9)$ & $1.6( \pm 1.0)$ & $3.0( \pm 1.3)$ & $1.5( \pm 0.9)$ \\
\hline 4 & $1,15( \pm 0.4)$ & $2.0( \pm 0.9)$ & $1.4( \pm 0.2)$ & $2.1( \pm 1.5)$ \\
\hline 6 & $2.4( \pm 0.5)$ & $4.5( \pm 0.8)$ & $3.7( \pm 0.4)$ & $2.3( \pm 1.2)$ \\
\hline 8 & $3.4( \pm 1.4)$ & $2.8( \pm 0.4)$ & $3.2( \pm 0.5)$ & $3.0( \pm 0.7)$ \\
\hline 10 & $3.4( \pm 0.9)$ & $4.0( \pm 1.1)$ & $2.7( \pm 0.2)$ & $2.6( \pm 0.3)$ \\
\hline 12 & $3.0( \pm 1.0)$ & $3.1( \pm 2.0)$ & $2.1( \pm 0.9)$ & $2.5( \pm 1.6)$ \\
\hline 14 & $2.0( \pm 1.1)$ & $4.4( \pm 2.7)$ & $2.9( \pm 1.4)$ & $3.0( \pm 0.3)$ \\
\hline 16 & $2.0( \pm 1.5)$ & $3.6( \pm 0.6)$ & $1.6( \pm 0.4)$ & $3.1( \pm 0.4)$ \\
\hline 19 & $2.3( \pm 1.5)$ & $1.3( \pm 0.2)$ & $1.1( \pm 0.9)$ & $2.8( \pm 0.4)$ \\
\hline \multicolumn{5}{|c|}{ Total Zooplankton (ind. $\mathrm{mL}^{-1}$ ) } \\
\hline 1 & $29200( \pm 1945)$ & $13183( \pm 3293)$ & $17967( \pm 7468)$ & $6600( \pm 3328)$ \\
\hline 2 & $4767( \pm 4544)$ & $1483( \pm 2569)$ & $19708( \pm 18083)$ & $19602( \pm 17491)$ \\
\hline 3 & $9883( \pm 4177)$ & $11150( \pm 4481)$ & $10433( \pm 2050)$ & $5950( \pm 2555)$ \\
\hline 4 & $9117( \pm 7425)$ & $21717( \pm 3201)$ & $12617( \pm 2470)$ & $6299( \pm 3593)$ \\
\hline 6 & $9800( \pm 7889)$ & $20800( \pm 6954)$ & $17450( \pm 3780)$ & $3425( \pm 895)$ \\
\hline 8 & $12561( \pm 9242)$ & $40114( \pm 24950)$ & $55367( \pm 8697)$ & $7117( \pm 3693)$ \\
\hline 10 & $15683( \pm 6634)$ & $70720( \pm 23138)$ & $69155( \pm 39674)$ & $6250( \pm 4653)$ \\
\hline 12 & $60438( \pm 30046)$ & $64625( \pm 31910)$ & $38367( \pm 40799)$ & $5263( \pm 2650)$ \\
\hline 14 & $34269( \pm 6361)$ & $92193( \pm 44661)$ & $158565( \pm 98687)$ & $14238( \pm 931)$ \\
\hline 16 & $85678( \pm 23525)$ & $9893( \pm 59667)$ & $316533( \pm 264486)$ & $99882( \pm 7406)$ \\
\hline 19 & $96736( \pm 101822)$ & $116224( \pm 73587)$ & $275561( \pm 151172)$ & $52196( \pm 17008)$ \\
\hline
\end{tabular}

na = data no avaiable.

the experiment and $2.9 \times 10^{4}$ cells $\mathrm{mL}^{-1}$ on the last day, and between $0.44 \times 10^{2}$ cells $\mathrm{mL}^{-1}$ on the $4^{\text {th }}$ day of experiment and $5.3 \times 10^{3}$ cells $\mathrm{mL}^{-1}$ on the $14^{\text {th }}$ experimental day. PNF density responded more quickly ( $1^{\text {st }}$ day after fertilization) and seems to have reached higher cell density in all nutrient addition treatments than did HNF. The time lag detected for HNF was of ca. 8 days after fertilization (Figure 2).

Furthermore, an interesting result concerning the pigmented fraction is the different patterns registered for $\mathrm{P}$ and $\mathrm{N}+\mathrm{P}$, with fast response (after Day $1^{\text {st }}$ ) and $N$ (important increase only after $6^{\text {th }}$ day; see the lowest $F$ estimated for the interaction) compared to the Control treatment. These and the PNF density response to nutrient addition were confirmed by the significant interactions among $\mathrm{N}$ $(F=2.3 ; p=0.020)$ and $\mathrm{P}(F=4 ; p=0.000)$ and the time of the experiment resulted from ANOVA.

\subsection{Nanoflagellate mean cell size}

In general, the mean body size of PNF nanoflagellates decreased in all treatments from the highest value on the $4^{\text {th }}$ day $(8.4 \mathrm{~mm})$ to minimal values $(2.41 \mathrm{~mm})$ on the $12^{\text {th }}$ day (Figure 3$)$. The results of the ANOVAR indicated significant effects of $\mathrm{N}(F=20.6 ; p=0.002), \mathrm{P}(F=2 ; p=0.015)$ and $\mathrm{N}+\mathrm{P}$ addition $(F=5.82 ; p=0.042)$. However, values varied through time $(F=3.63 ; p=0.001)$.

Within the heterotrophic fraction, we observed mean cell sizes from $8.4 \mu \mathrm{m}$ on the $3^{\text {rd }}$ day to $2.41 \mu \mathrm{m}$ on the $19^{\text {th }}$ day. However, unlike in the pigmented fraction, a clear trend was not observed for the cell size changes (Figure 3). The ANOVAR showed a significant effect of $\mathrm{N}$-addition $(\mathrm{F}=6.85$; $\mathrm{p}=0.034)$ and a variation of values with time in fertilized treatments $(\mathrm{F}=2.9 ; \mathrm{p}=0.003)$.

\subsection{Nanoflagellate biomass}

Pigmented total biomass increased from the initial value of $0.33 \mathrm{mg} \mathrm{C} \mathrm{L}^{-1}\left(1^{\text {st }}\right.$ day) to $102.9 \mathrm{mg}$ $\mathrm{C} \mathrm{L} \mathrm{L}^{-1}$ on the last day. The HNF biomass on the $3^{\text {rd }}$ day $\left(0.21 \mathrm{mg} \mathrm{C} \mathrm{L}^{-1}\right)$ increased to $173 \mathrm{mg} \mathrm{C} \mathrm{L}^{-1}$ on the 14 days. Moreover, a time lag in the response of nanoflagellate biomass increase was observed. In $\mathrm{N}+\mathrm{P}$-addition, the initial increase occurred on the $1^{\text {st }}$ day after fertilization (Figure 4).

The effects of $\mathrm{P}$ and $\mathrm{N}$ addition on the PNF biomass fraction were significant but varied through 

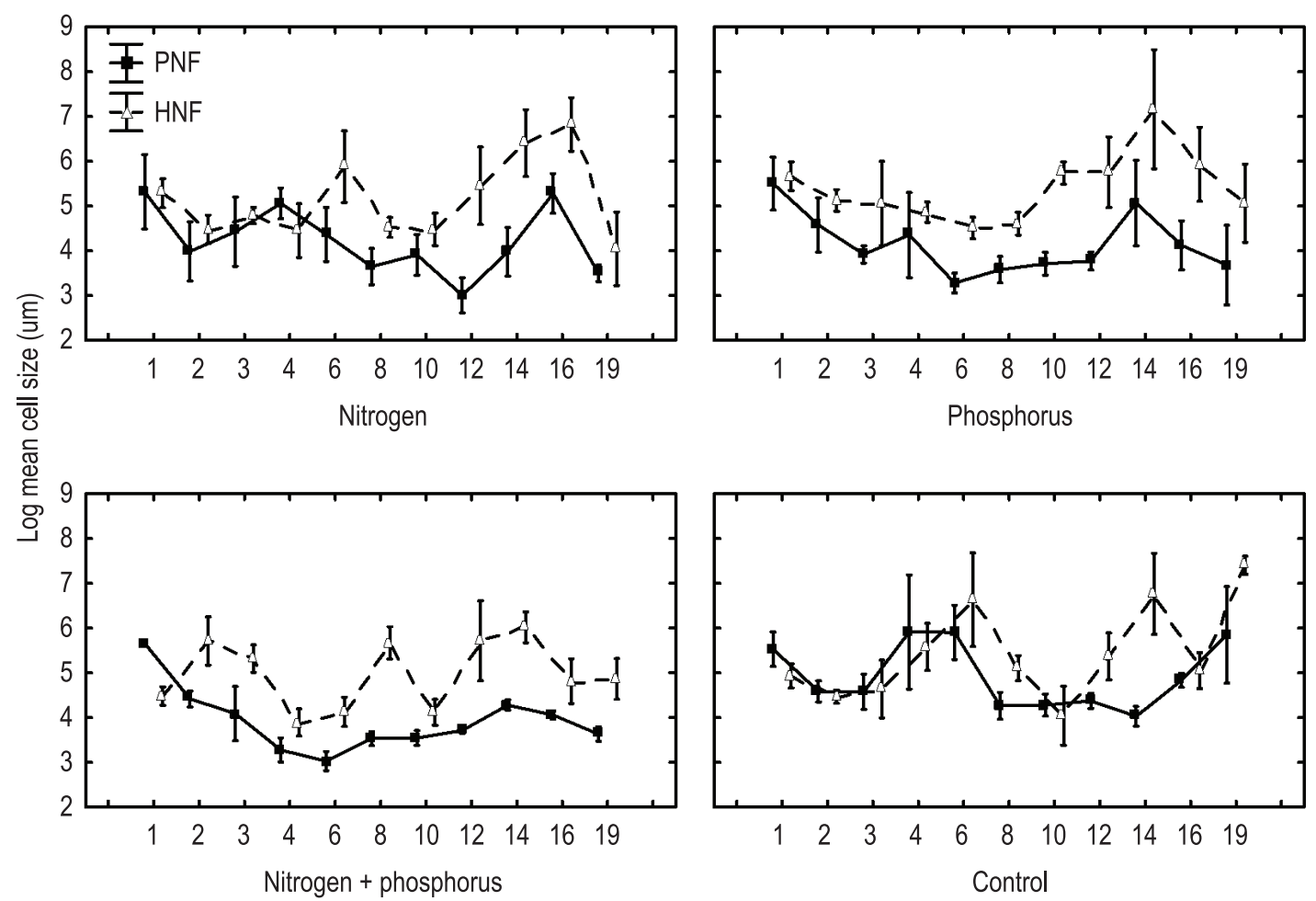

Days

Figure 3. Variation of pigmented (PNF) and heterotrophic (HNF) nanoflagellate mean cell size during 19 days of a nutrient addition experiment. Data shown are mean \pm standard deviation.

time, as indicated by the interactions $(F=3.3$; $p=0.001$ and $\mathrm{F}=2.2 ; \mathrm{p}=0.026$ for $\mathrm{P}$ and $\mathrm{N}$ along the time, respectively). On the other hand, no significant response of HNF biomass to nutrient enrichment was detected.

\subsection{PNF: HNF ratio}

Nutrient enrichment caused an increase in the ratio of PNF to HNF density. The greatest difference between fractions occurred when both $\mathrm{P}$ and $\mathrm{N}$ were added (Figure 2). Moreover, the effect of $\mathrm{N}$-alone and $\mathrm{P}$-alone addition varied along the time as indicated by the significant interactions among these treatments and time $(F=1.996 ; p=0.044$; $F=3.510 ; p=0.001)$.

The effect of nutrient addition on the ratio PNF: HNF biomass suggested an effect only with regard to the $\mathrm{N}+\mathrm{P}$-addition treatment, where a temporal increase of the PNF contribution was observed (Figure 4). However, significant effects of nutrient addition were not observed, with only significant changes in biomass values along the time $(F=2.30$; $p=0.020)$.

\section{Discussion}

The density, biomass, and mean cell size of the nanoflagellate community were affected by nutrient addition, paralleling results obtained in several experimental studies (Jansson et al., 1996; Gilbert et al., 1998; Simek et al., 2003). The increase in the abundance of PNF and HNF as a response to the increase in nutrient availability is also frequently reported in non-manipulative studies (Gasol et al., 1995; Hwang and Heath, 1997; Hobbie et al., 1999; Auer and Arndt, 2001; Samuelsson et al., 2002, 2006; Auer et al., 2004) showing the influence of the lake trophy on the abundance and biomass of distinct compounds of the planktonic food web. Studies have shown that the increase in availability of resources in the environment affects the propagation of all components of the planktonic food web, evidencing the relevance of the bottom up control mechanism (Andersson et al., 2006).

Concerning to the cell size, our results differ from the general trend of a positive relationship between resources and mean cell size (Kress et al., 2005; Sabetta et al., 2005; Sipura et al., 2005; Samuelsson et al., 2006). Small organisms are 

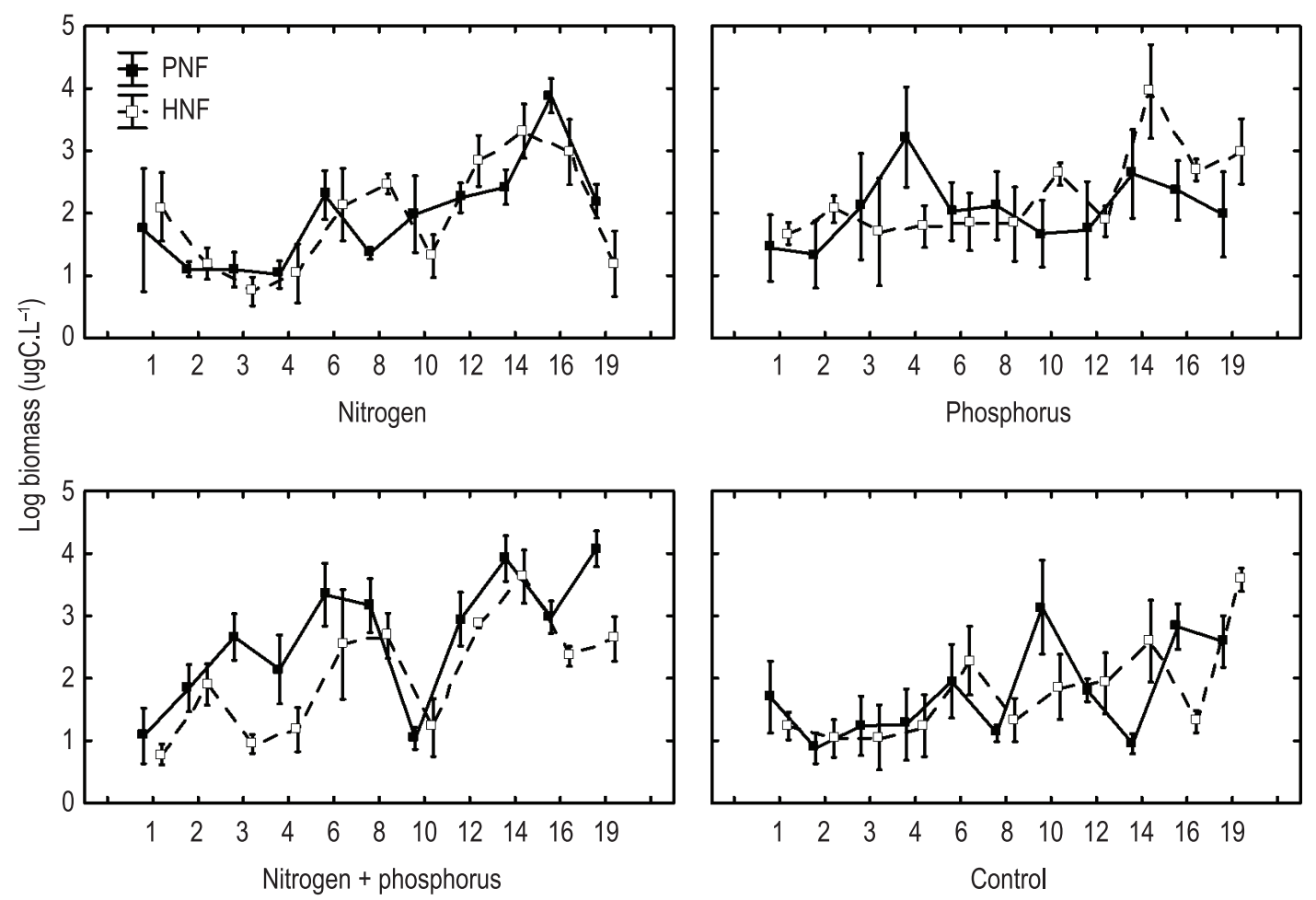

Days

Figure 4. Variation of pigmented (PNF) and heterotrophic (HNF) nanoflagellate biomass during 19 days of a nutrient addition experiment. Data shown are mean \pm standard deviation.

supposedly better adapted to exploit resources in lower concentrations (Berninger and Wickham, 2005) due to their higher surface:volume ratio, which allows a rapid utilization of resource (Pirlot et al., 2005) and provides competitive advantage in oligotrophic environments.

The significant decrease of PNF mean cell size in fertilized conditions registered in the present study was also observed by Racy (2004), studying the bacterial community in a tropical reservoir with different trophic scales. This author suggested that greater densities observed in eutrophic environments would result from an increase in reproductive rate by cell division, leading to a decrease in mean body size.

Another factor that could be related to the decrease of PNF mean cell size relates to top down control mechanisms. The increase in predator density caused by propagation of bottom-up effect, at all levels of the food web after nutrient fertilization, results in higher predation pressure on large bodied nanoflagellates, and a subsequent decrease in mean body size for this community is expected. According to Samuelsson and Andersson (2003), predation pressure seems to be size-dependent, increasing with the body size of nanoflagellates.

Considering the short generation time (hours), and the high growth rate of nanoflagellates (1 to 5 times/day in good growth conditions) (LaybournParry, 1992), these organisms respond with a rapid increase in abundance after nutrient fertilization (e.g Burns and Schallenberg, 1998). However, we observed time lag differences of PNF and HNF abundances. The PNF tended to respond faster than HNF because they have the capacity to assimilate the nutrients directly from the environment while the HNF incorporate nutrients mainly through bacteria and autotrophic nanoflagellates, and therefore responded indirectly to the nutrient fertilization.

In our study, the faster and greater amplitude of response observed in treatments fertilized with phosphorus (P-alone addition), over those fertilized with nitrogen ( $\mathrm{N}$-alone addition), seemed to corroborate the hypothesis that phosphorus is the main limiting factor for the growth of freshwater aquatic communities. However, a similar trend of abundance increase was observed in the $\mathrm{N}$ addition treatment, although at a lower scale and later (after the $4^{\text {th }}$ day) than in the $\mathrm{P}$ addition, thus arguing with the idea of co-limitation of $\mathrm{N}$ and $\mathrm{P}$ suggested by Huszar (2006).

The nearly 1:1 relative contribution of autotrophic and heterotrophic fractions to the total 
density and biomass of nanoflagellates observed in controlled experimental units and on the first days in fertilized ones has not been often recorded. Conversely, it has been registered the predominance of autotrophic organisms in natural environments (Safi and Hall, 1997) and independent of trophic state (Samuelsson et al., 2002).

In summary, our results indicate that: i) the pigmented and heterotrophic fractions showed distinct time responses to fertilization ii) the growth of nanoflagellate community seems to be co-limited by $\mathrm{N}$ and $\mathrm{P}$; iii) the nutrient enrichment led to a greater pigmented than heterotrophic fraction contribution; and v) among the analyzed attributes, nanoflagellate densities seem to be more sensitive to changes in nutrient availability than biomass or mean body size.

\section{References}

ANDERSSON, A. SAMUELSSON, K., HAECKY, P. and ALBERTSSON, J. 2006. Changes in the pelagic microbial food web due to artificial eutrophication. Aquatic Ecology, vol. 40, p. 299-313. http://dx.doi. org/10.1007/s10452-006-9041-7

AUER, B., and ARNDT, H. 2001. Taxonomic composition and biomass of heterotrophic flagellates in relation to lake trophy and season. Freshwater Biology, vol. 46, p. 959-972. http://dx.doi. org/10.1046/j.1365-2427.2001.00730.x

AUER, B., ELZER, U. and ARNDT, H. 2004. Comparision of pelagic food webs in lakes along a trophic gradient and with seasonal aspects: influence of resource and predation. Journal of Plankton Research, vol. 26, p. 697-709. http://dx.doi. org/10.1093/plankt/fbh058

BERGAMIN, H., REIS, BF. and ZAGATTO, EAG. 1978. A new device for imptoving sensitivity and stabilization in inflow injection analysis. Analytica Chimica Acta, vol. 97, p. 63-70.

BERNINGER, U-G. and WICKHAM, SA. 2005. Response of the microbial food web to manipulation of nutrients and grazers in the oligotrophic Gul of Aqaba and northen Red Sea. Marine Biology, vol. 147, p. 1017-1032. http://dx.doi.org/10.1007/s00227005-1565-1

BOTTRELL, HH., DUNCAN, A., GLIWICZ, Z., GRYGIEREK, E., HERZIG, A., HILLBRICHTILLKOWSKA, A., KURASAWA, H., LARSSON, P. and WEGLENSKA, T. 1976. A review of some problems in zooplankton production studies. Norwegian Journal of Zoology, vol. 24, p. 419-456.

BURNS, CW. and SCHALLENBERG, M. 1998. Impacts of nutrients and zooplankton on the microbial food web of an ultra-oligotrophic lake. Jounal of Plankton Research, vol. 20, p. 1501-1525.
CARVALHO, P., THOMAZ, SM. and BINI, LM. 2003. Effects of water level, abiotic and biotic factors on bacterioplankton abundance in lagoons of a tropical floodplain (Paraná River, Brazil). Hydrobiologia, vol. 510, p. 67-74. http://dx.doi.org/10.1023/ B:HYDR.0000008532.71152.38

DOMÈNECH, R., GAUDES, A., LÓPEZ-DOVAL, JC., SALVADO, H. and MUHOZ, I. 2006. Effects of short-term nutrient addition on microfauna density in a Mediterranean stream. Hydrobiologia, vol. 568, p. 207-215. http://dx.doi.org/10.1007/ s10750-006-0107-2

FENCHEL, T. 1982. Ecology of heterotrophic flagellates. IV. Quantitative occurrence and importance as bacterial consumers. Marine Ecology Progress Series, vol. 9, p. 35-42. http://dx.doi.org/10.3354/ meps009035

GASOL, JM., SIMONS, AM. and KALFF, J. 1995. Patterns in the top-down vesus bottom-up regulation of heterotrophic nanoflagellates in temperate lakes. Jounal of Plankton Research vol. 17, p. 1879-1903. http://dx.doi.org/10.1093/plankt/17.10.1879

GILBERT, D., AMBLARD, C., BOURDIE, G. and FRANCEZ, A-J. 1998. The microbial loop at the surface of a peatland: Structure, Function, and impact of nutrient input. Microbial Ecology, vol. 35, p. 8393. PMid:9459661. http://dx.doi.org/10.1007/ s002489900062

GOLTERMAN, HL, CLYMO, RS. and OHMSTAD, MAM. 1978. Methods for physical and chemical analysis of freshwater. Oxford: Blackwell Scientific Publication. 214 p.

HOBBIE, JE., BAHR, M. and RUBLEE, PA. 1999. Controls on microbial food webs in oligotrophic artic lakes. Archiv für Hydrobiologie Special Issues Advanced Limnology, vol. 54, p. 61-76.

HUSZAR, VLM., CARACO, NF., ROLAND, F. and COLE, J. 2006. Nutrient-chlorophyll relationships in tropical-subtropical lakes: do temperate models fit? Biogeochemistry, vol. 79, p. 239-250. http://dx.doi. org/10.1007/s10533-006-9007-9

HWANG, SJ. and HEALTH, RT. 1997. The distribution of protozoa across a trophic gradient, factors controlling their abundance and importance in the plankton food web. Journal of Plankton Research, vol. 19, p. 491-518. http://dx.doi.org/10.1093/ plankt/19.4.491

JANSSON, M. 1996. Nutrient limitation of bacterioplankton, autotrophic and mixotrophic phytoplankton, and heterotrophic nanoflagellates in Lake Ortrasket. Limnology and Oceanography, vol. 4, p. 1552-1559. http://dx.doi.org/10.4319/ lo.1996.41.7.1552

KRESS, N., THINGSTAD, TF., PITTA, P., PSARRA, S., TANAKA, T., ZOHARY, T., GROOM, S., HERUT, B., MANTOURA, RFC., POLYCHRONAKI, T., 
RASSOULZADEGAN, F. and SPYRES, G. 2005. Effect of $\mathrm{P}$ and $\mathrm{N}$ addition to oligotrophic Eastern Mediterranean waters influenced by near-shore waters: A microcosm experiment. Deep-Sea Research Part II, vol. 52, p. 3054-3073.

LAMPERT, W. and SOMMER, U. 1997. Limnoecology: The ecology of lakes and streams. New York: Oxford University Pres. $382 \mathrm{p}$.

LAYBOURN-PARRY, J. 1992. Protozoan plankton ecology. Chapman and Hall, London: $238 \mathrm{p}$.

LEWIS JUNIOR, WM. 2000. Basis for the protection and management of tropical lakes. Lake and Reservoir Managements, vol. 5, p. 35-48. http://dx.doi. org/10.1046/j.1440-1770.2000.00091.x

MacKERETH, FYH. 1978. Water analysis: some revised methods for limnologists. Freshwater Biology Association, vol. 36, p. 1-120.

PAGIORO, TA., VELHO, LFM., LANSACTÔHA, FA., PEREIRA, DG. and NAKAMURA, AK. 2005. Influência do grau de trofia sobre os padróes de abundância de bactérias e protozoários planctônicos em reservatórios do Estado do Paraná. In RODRIGUES, L., THOMAZ, SM., AGOSTINHO, AA. and GOMES, LC., org. Biocenoses em reservatórios: Padróes espaciais e temporais. São Carlos: RIMA. p. 37-46.

PIRLOT, S., VANDERHEYDEN, J., DESCY, J-P. and SERVAIS, P. 2005. Abundance and biomass of heterotrophic microorganisms in Lake Tanganyika. Freshwater Biology, vol. 50, p. 1219-1232. http:// dx.doi.org/10.1111/j.1365-2427.2005.01395.x

RACY, FPP. 2004. Aspectos numéricos, morfológicos, e morfométricos da comunidade bacteriana em diferentes escalas trófica e temporal em reservatórios. São Carlos: Universidade Federal de São Carlos. [Dissertação em Ecologia e Recursos Naturais].

REJAS, D., MUYLAERT, K. and DE MEESTER, L. 2005. Trophic interactions within the microbial food web in a tropical floodplain lake (Lguna Bufeos, Bolivia) Revista de Biología Tropical, vol. 53, p. 1-2.

ROBERTO, MC., THOMAZ, SM. and PAGIORO, TA. 2005. Variáveis Limnológicas Abióticas: Caracterização dos Reservatórios. In RODRIGUES, L., THOMAZ, SM., AGOSTINHO, AA. and GOMES, LC., org. Biocenoses em reservatórios: Padróes espaciais e temporais. São Carlos: RIMA. p. $19-25$

SABETTA, L., FIOCCA, A., MARGHERITI, L., VIGNES, F., BASSET, A., MANGONI, O., CARRADA, GC., RUGGIERI, N. and IANNI,
C. 2005. Body size-abundance distributions of nanoand micro-phytoplankton guilds in coastal marine ecosystems. Estuarine, Coastal and Shelf Science, vol. 63, p. 645-663. http://dx.doi.org/10.1016/j. ecss.2005.01.009

SAFI, KA. and HALL, JA. 1997. Factors influencing pigmented and heterotrophic nanoflagellate abundance in five water masses surrouding New Zealand. New Zealand Journal of Marine \& Freshwater Research, vol. 31, p. 51-60. http://dx.doi.org/10.108 0/00288330.1997.9516744

SAMUELSSON, K., BERGLUND, J., HAECKY, P. and ANDERSON, A. 2002. Structural changes in an aquatic microbial food web caused by inorganic nutrient addition. Aquatic Microbial Ecology, vol. 29, p. 29-38. http://dx.doi.org/10.3354/ame029029

SAMUELSSON, K. and ANDERSSON, A. 2003. Predation limitation in the pelagic microbial food web in an oligotrophic aquatic system. Aquatic Microbial Ecology, vol. 30, p. 239-250. http://dx.doi. org/10.3354/ame030239

SAMUELSSON, K., BERGLUND, J. and ANDERSON, A. 2006. Factors structuring the heterotrophic flagellate and ciliate community along a brackish water primary production gradient. Journal of Plankton Research, vol. 28, p. 345-359. http://dx.doi. org/10.1093/plankt/fbi118

SHERR, EB. and SHERR, BF. 1993. Preservation and storage of samples for enumeration of heterotrophic protists. In KEMP, PF., SHERR, BF., SHERR, EB. and COLE, JJ., eds. Handbook of methods in Aquatic Microbial Ecology. London: Lewis Publishers. p. 207-212.

SIMEK, K., HORNAK, K., MASIN, M., CHRISTAK, U., NEDOMA, J., WEINBAUERN, MG. and DOLAN, JR. 2003. Comparing the effects of resource enrichiment and grazing on a bacterioplankton community. Aquatic Microbial Ecology, vol. 31, p. 123-135. http://dx.doi.org/10.3354/ame031123

SIPURA, J., HAUKKA, K., HELMINEN, H., LAGUS, A., SUOMELA, J. and SIVONEN, K. 2005. Effect of nutrient enrichment on bacterioplankton biomass and community composition in mesocosms in the Archipelago Sea, northen Baltic. Journal of Plankton Research, vol. 27, p.1261-1272. http://dx.doi. org/10.1093/plankt/fbi092

Statsoft Inc., 1997. Statistica for windows (Computer program manual). Tulsa Oklahoma.

WETZEL, RG. and LIKENS, GE. 1991. Limnological Analyses. New York: Springer-Verlag. 391 p. 